\title{
DNA fingerprints of anaerobic fungi
}

\author{
K Fliegerova, S Pazoutova, V Kostyukovsky, J Kopecny \\ 1/nstitute of Animal Physiology and Genetics, Czech Academy of Sciences, Prague 10, Uhrineves, \\ 2Institute of Microbiology, Czech Academy of Sciences, Prague 4, Krc, Czech Republic
}

The anaerobic fungi represent a special group of microorganisms inhabiting the rumen ecosystem. They are recognised as the producers of the most effective polysaccharides. All rumen fungi have been assigned to the family of the Neocallimasticaceae of the class Chytrdiomycetes. Within this family six genera have been recognized: Neocallimastix, Piromyces, Caecomyces, Orpinomyces, Anaeromyces and Ruminomyces. These genera can be partially characterized on the basis of thallus morphology and the ultrastructure of their zoospores. The main problem in the systematics of the Chytridiomycetes is that many species display extensive morphological variation depending on the environmental conditions. Molecular techniques offer a new approach to systematics. Information at the genetic level could give us a powerful tool for assessing phylogenetic relationships. Restriction fragment length polymorphisms (RFLP) can be used as one of several molecular methods to study of evolutionary relationships and identification of fungi. The proportion of bands common to the DNA of isolates in RFLP analysis is measure of similarity between the isolates.
In our preliminary experiments we attempted to apply RFLPs to four isolates of anaerobic fungi: Neocallimastix frontalis KR3 (from rumen of Bos taurus), Caecomyces communis L2 (from feces of Lamma paca), Orpinomyces joyonii A4 (from rumen of Camelus bactrianus) and Zu1 (from feces of Bibos bonasus). Their genomic DNAs were isolated according to the method of Brownlee (1989, Nucl Acids Res, 17, 1327-1335) and cleaved by EcoRI enzyme. Digested DNAs were separated in agarose gel $(0.7 \%, 5 \mathrm{v} / \mathrm{cm})$ in TBE buffer. After electrophoresis EcoRIdigested nuclear DNAs were transfered to membrane and non-radioactively hybridized with $\mathrm{RS} 2$ repetitive sequence ((GAC)5) (Rodriguez and Yoder, 1991, Exp Mycol, 15, 232-242).

The EcoRI restriction pattern of genomic DNAs on gel electrophoresis showed differences among the four fungi. Hybridization experiments revealed that repetitive element RS2 hybridizes only to DNA of Caecomyces communis L2 (one strong band-3.7 kpb). The fungi showed completely different in banding pattern with RS2. Strains KR3 and A4 did not hybridize at all. Isolates do not share any similarities based on RFLPs. 